\title{
Aircraft parameter estimation - A tool for development of aerodynamic databases
}

\author{
R V JATEGAONKAR and F THIELECKE
}

Institute of Flight Mechanics, German Aerospace Center (DLR), Lilienthalplatz 7, 38108 Braunschweig, Germany

e-mail: \{jategaonkar, frank.thielecke $\} @$ dlt.de

\begin{abstract}
With the evolution of high performance modern aircraft and spiraling developmental and experimental costs, the importance of flight validated databases for flight control design applications and for flight simulators has increased significantly in the recent past. Ground-based and in-flight simulators are increasingly used not only for pilot training but also for other applications such as flight planning, envelope expansion, design and analysis of control laws, and handling qualities investigations. Most of these demand a high-fidelity aerodynamic database representing the flight vehicle. System identification methodology, evolved over the past three decades, provides a powerful and sophisticated tool to identify from flight data aerodynamic characteristics valid over the entire operational flight envelope. This paper briefly presents aircraft parameter estimation methods for both stable and unstable aircraft, highlighting the developmental work at the DLR Institute of Flight Mechanics. Various aspects of database identification and its validation are presented. Practical aspects like the proper choice of integration and optimization methods as well as limitations of gradient approximation through finite-differences are brought out. Though the paper focuses on application of system identification methods to flight vehicles, its use in other applications, like the modelling of inelastic deformations of metallic materials, is also presented. It is shown that there are many similar problems and several challenges requiring additional concepts and algorithms.
\end{abstract}

Keywords. Aircraft parameter estimation; aerodynamic databases; system identification.

\section{Introduction}

Full-fledged flight crew training simulators for commercial transport as well as high performance aircraft, in general, should demonstrate high fidelity, often meeting the Level D fidelity standards specified by FAA, the Federal Aviation Administration (Anon 1991, 1995). Fidelity of flight simulation depends to a large extent on the accuracy of the aerodynamic database representing the aircraft. Furthermore, the demands of high-performance characteristics have led to aerodynamically unstable aircraft configurations, which can be flown only with the aid of flight controllers based on modern control concepts. In this case, 
it is mandatory that the aerodynamic characteristics used for control law design are representative of the actual aircraft.

To minimize the overall developmental costs, it is necessary to minimize the amount of costly and time-consuming flight testing necessary for aircraft specification and certification requirements. Furthermore, the new aerodynamic design concepts such as, for example, thrust vectoring (Ross 1991) or quasi-tailless aircraft (Huber 1995), require careful expansion of the flight envelope through (i) data correlation between the prediction and flight identified aerodynamic characteristics, and (ii) isolation and identification of non-anticipated aerodynamic effects. This is also true in the case of large flexible aircraft, where the structural modes can influence the aircraft response and the accuracy of flight mechanical models (Chevalier et al 1968; Rynaski et al 1978). The philosophy of model-following control based on feed forward regulation provides safer and more accurate mode control; performance, however, depends strongly on the validated mathematical model of the host flight vehicle (Hamel \& Kaletka 1995). For such an application, the rotorcraft flight control has to meet the high bandwidth requirements, demanding augmentation of the rigid-body model through higher-order rotor dynamics.

In the majority of the cases an aerodynamic database derived from analytical predictions, wind tunnel measurements on a scaled model or extrapolation of existing data from similar configurations is incorporated. Such databases, although valid over the entire flight envelope, are often associated with certain limitations of validity arising from, for example, model scaling, Reynold's number, dynamic derivative, and cross coupling effects (Hamel 1981). On the other hand, for each of the aforementioned flight vehicle applications, it is possible to obtain homogeneous, high-fidelity aerodynamic databases applying the coordinated approach of flight vehicle system identification. Availability of a flexible and sophisticated parameter estimation tool is, however, a pre-requisite for such advanced applications. Systematic developmental work at the DLR Institute of Flight Mechanics has resulted in a comprehensive and flexible aircraft parameter estimation software that caters to linear or nonlinear systems with measurement as well as process noise, and to stable as well as unstable plants, offers a wide variety of integration methods to suit different dynamic systems, and also a large number of optimization methods starting from the simplex search method to more advanced methods such as the Gauss-Newton or LevenbergMarquardt method. Some typical examples which bring out the characteristic features of the aircraft parameter estimation software are presented.

In general, aircraft motion represents a highly dynamic system with relatively low time constants and observation durations. On the other hand, the viscoplastic behaviour of metals like pure aluminum, pure copper or austenitic steel AISI 316 represents characteristically different dynamic systems having very large observation times and slowly changing dynamic systems, in many cases with stiff differential equations. Since replicated experiments for the same laboratory conditions show a significant scattering, these uncertainties must be taken into account for parameter estimation. Applicability of parameter estimation methods to such problems is also presented in this paper.

\section{Estimation methods}

For conventionally stable flight vehicles, the most commonly and widely applied parameter estimation methods are: (i) regression analysis and (ii) output error method. Regression analysis is based on linear models for the aerodynamic phenomena and requires error-free 
and compatible measurements. The output error method, which has several desirable statistical properties, is applicable to general nonlinear systems and accounts for measurement noise (Maine \& Iliff 1986; Klein 1989; Hamel \& Jategaonkar 1996).

The dynamic system, whose parameters are to be estimated, is assumed to be described in state space as:

$$
\begin{aligned}
\dot{x}(t) & =f[x(t), u(t), \beta], \quad x\left(t_{0}\right)=x_{0}, \\
y(t) & =g[x(t), u(t), \beta], \\
z\left(t_{k}\right) & =y\left(t_{k}\right)+v\left(t_{k}\right),
\end{aligned}
$$

where $x$ is the $n$-dimensional state vector, $u$ is the $p$-dimensional control input vector, $y$ is the $m$-dimensional observation vector, and $z$ is the output vector, i.e., the discrete measurement of $y$. The system functions $f$ and $g$ are general nonlinear real-valued vector functions. The measurement noise $v$ is assumed to be characterized by a sequence of independent Gaussian random variables with zero mean and covariance matrix $R$. The goal is to estimate the unknown system parameters $\beta$ from the discrete measurements of the system response $z\left(t_{k}\right)$ to the given inputs $u\left(t_{k}\right)$ based on the mixed continuous/discrete system model postulated in (1)-(3). In addition to the unknown system parameters $\beta$, the initial conditions $x_{0}$ are also unknown. Furthermore, the measurements of $z$ and $u$ are likely to contain systematic errors $\Delta z$ and $\Delta u$ respectively. In a general case, the unknown parameter vector $\Theta$ to be estimated is given by:

$$
\Theta=\left[\beta^{T} ; x_{0}^{T} ; \Delta z^{T} ; \Delta u^{T}\right] .
$$

The maximum likelihood estimates of the unknown parameters are obtained by minimization of the negative log likelihood function:

$$
J(\Theta, R)=\frac{1}{2} \sum_{k=1}^{N}\left[z\left(t_{k}\right)-y\left(t_{k}\right)\right]^{T} R^{-1}\left[z\left(t_{k}\right)-y\left(t_{k}\right)\right]+\frac{N}{2} \ln |R| .
$$

Starting from the specified initial values of $\Theta$, new updated estimates are obtained by applying the Gauss-Newton method:

$$
\begin{aligned}
\Theta_{i+1} & =\Theta_{i}+\Delta \Theta_{i}, \\
\Delta \Theta & =\left\{\sum_{k=1}^{N}\left[\frac{\partial y\left(t_{k}\right)}{\partial \Theta}\right]^{T} R^{-1}\left[\frac{\partial y\left(t_{k}\right)}{\partial \Theta}\right]\right\}^{-1}\left\{\sum_{k=1}^{N}\left[\frac{\partial y\left(t_{k}\right)}{\partial \Theta}\right]^{T} R^{-1}\left[z\left(t_{k}\right)-y\left(t_{k}\right)\right]\right\} .
\end{aligned}
$$

The first term in braces on the right-hand side of (7) is an approximation of the second gradient $\left[\partial^{2} J / \partial^{2} \Theta\right]$. This approximation helps to reduce the computational costs without significantly affecting the convergence.

The iterative update of $\Theta$ using (6) and (7) thus requires: (i) computation of the observation variables $y$, and (ii) computation of the response gradients $[\partial y / \partial \Theta]$ at each time point $t_{k}$. Efficient implementation of these computational aspects, together with flexibility to handle conveniently different model structures, is important.

Computation of the observation variables requires computation of state variables. For linear systems the state transition matrix provides efficient solution. However, since the primary interest is in the nonlinear systems, it becomes mandatory that some suitable numerical integration method be used. The response gradients $[\partial y / \partial \Theta]$ can be evaluated 
using either the analytical differentiation or by finite difference approximation. In the case of linear systems, the analytical differentiation resulting in explicit sensitivity equations is usually employed. For nonlinear systems, the analytical differentiation is tedious and impracticable, requiring model-dependent changes anytime the postulated model is changed. Hence, finite difference approximation is usually employed. The gradients can be approximated using the forward difference approximation as:

$$
\frac{\partial y}{\partial \Theta} \approx \frac{y_{p}(\Theta+\delta \Theta)-y(\Theta)}{\delta \Theta},
$$

where $y_{p}$ is the perturbed response vector computed from (2) using the perturbed state obtained by integrating the state equations for the small perturbation $\delta \Theta$. Alternatively, the gradients can be computed through central difference approximation as:

$$
\frac{\partial y}{\partial \Theta} \approx \frac{y_{p}^{+}(\Theta+\delta \Theta)-y_{p}^{-}(\Theta-\delta \Theta)}{2 \delta \Theta} .
$$

The accuracy of the forward and central difference approximations is of the order of $O(h)$ and $O\left(h^{2}\right)$ respectively, where $h$ is the perturbation. The central difference approximation requires twice the number of evaluations, which can amount to significant computational overload, particularly when large problems are addressed. These numerical aspects, namely the choice of suitable integration method and accuracy of approximated gradients need some consideration.

It is well-known that the Gauss-Newton method has a quadratic convergence within a certain region around the minimum. However, a bad guess of the initial parameter values may lead to convergence difficulties resulting from errors in the approximation of the Hessian. In such cases the Levenberg-Marquardt method, which combines the features of the Gauss-Newton and steepest descent method and thereby has a wider convergence region, may be preferable (Marquardt 1963). Investigations, however, showed that a special startup procedure using least squares is more efficient. This regression startup, however, requires measurements of the state variables, which may be a limitation in certain applications (Jategaonkar \& Thielecke 1994). The Levenberg-Marquardt update formula is given by:

$$
\Delta \Theta=(F+\lambda I)^{-1} G,
$$

where $F$ and $G$ are the information matrix, i.e., approximation to the Hessian, and gradient given by the first term and second term on the right hand side of (7) respectively, and $\lambda$ is the Levenberg-Marquardt parameter. The adaptation parameter $\lambda$ is decreased or increased from iteration to iteration depending upon whether the optimization progresses in the Gauss-Newton direction monotonically or not.

\section{Practical aspects of numerical computations}

The choice of the integration method depends on the type of the system being investigated. Although, the fourth-order Runge-Kutta method is generally recommended for rigid-body aircraft dynamics, this choice may be more conservative. As a typical example, estimation of derivatives pertaining to the longitudinal motion from a two-point aerodynamic model accounting for wing-body and tail separately is considered (Jategaonkar et al 1994). As seen from table 1, second- and third-order methods yield equally acceptable results 
Table 1. Estimates of aerodynamic derivatives applying different integration methods.

\begin{tabular}{lccccc}
\hline & \multicolumn{5}{c}{ Method } \\
\cline { 2 - 6 } & Euler & $\begin{array}{c}\text { Second order } \\
\text { Runge-Kutta }\end{array}$ & $\begin{array}{c}\text { Third order } \\
\text { Runge-Kutta }\end{array}$ & $\begin{array}{c}\text { Fifth order } \\
\text { Fourth order } \\
\text { Runge-Kutta }\end{array}$ & $\begin{array}{c}\text { Runge-Kutta- } \\
\text { Fehlberg }\end{array}$ \\
\hline$C_{\mathrm{L} 0}$ & 0.0682 & 0.0770 & 0.0775 & 0.0775 & 0.0775 \\
$C_{\mathrm{D} 0}$ & 0.0232 & 0.0296 & 0.0297 & 0.0297 & 0.0297 \\
$C_{m 0}$ & -0.1732 & -0.1731 & -0.1732 & -0.1732 & -0.1732 \\
$C_{\mathrm{L} \alpha}$ & 5.536 & 5.443 & 5.437 & 5.438 & 5.437 \\
$e$ & 0.646 & 1.038 & 1.049 & 1.048 & 1.048 \\
$C_{\mathrm{L} \delta e}$ & 1.436 & 1.427 & 1.433 & 1.434 & 1.433 \\
$C_{m q W B}$ & 10.362 & 11.39 & 11.23 & 11.23 & 11.23 \\
Det (R) & $2.32 \times 10^{-9}$ & $1.25 \times 10^{-9}$ & $1.25 \times 10^{-9}$ & $1.25 \times 10^{-9}$ & $1.25 \times 10^{-9}$ \\
Iterations & 7 & 5 & 5 & 5 & 5 \\
\hline
\end{tabular}

compared to the more time-consuming fourth- and fifth-order Runge-Kutta formulae. The Euler integration, although it appears to converge to almost the same minimum, does show some changes in the numerical values, and convergence is affected as evident from the increase in the number of iterations. Moreover, the performance deteriorates for sampling times greater than 20 milliseconds and propagation of errors is unacceptable, particularly when the flight maneuver is of longer duration. For systems characterized by higher order dynamics, integration methods with step size control will be necessary to ensure that errors are low and that they do not propagate adversely, which may affect the accuracy of the estimates and convergence of the optimization method.

In the case of the Gauss-Newton method incorporating finite-difference approximation, the accuracy of the approximated gradients becomes critical near the minimum. For a typical example, table 2 shows the convergence of the Gauss-Newton method for the two cases of approximating the gradients through forward and central difference. For the forward difference case, the optimization progresses monotonically for the first eight iterations, and thereafter the 9th and 10th iterations encounter divergence which can be corrected either through line search or through heuristic procedure of halving the parameter update. In both these versions of line search or heuristic correction, the search direction remains unchanged. In the following iteration, the optimization procedure was terminated because no further improvement in the cost function was possible. On the other hand, for the second case in which the gradients are approximated through central difference, the convergence is monotonic and proper termination to the desired accuracy is achieved after nine iterations. The difference in the cost function value for the two cases and the difficulties in the progress of optimization in the first case are demonstrative of the limitations of the finite-difference approximation used in gradient computations.

The results for the same example obtained by applying the Levenberg-Marquardt method are also presented in table 2. In this case, it is observed that the optimization progresses without any difficulty for the first seven iterations using the Gauss-Newton directions. Subsequently, however, the Gauss-Newton directions computed using the forwarddifference approximation tend to be inaccurate, and by adjusting the adaptation factor $\lambda$ the method tends to choose the gradient-like directions. This property of detecting the divergence due to inaccuracies and then progressing in the gradient-like direction ensures reduction in the cost function from iteration to iteration, but the slow progress, as evident from the several iterations required, is a typical characteristic of the steepest descent method. 
Table 2. Performance of finite-difference approximations, Gauss-Newton and LevenbergMarquardt methods.

\begin{tabular}{lccc}
\hline Iteration & $\begin{array}{c}\text { Gauss-Newton with } \\
\text { forward difference } \\
\text { approximation }\end{array}$ & $\begin{array}{c}\text { Gauss-Newton with } \\
\text { central difference } \\
\text { approximation }\end{array}$ & $\begin{array}{c}\text { Levenberg-Marquardt with } \\
\text { forward difference } \\
\text { approximation }\end{array}$ \\
\hline 0 & $3.954 \mathrm{D}+01$ & $3.9542 \mathrm{D}+01$ & $3.9542 \mathrm{D}+01$ \\
1 & $4.9375 \mathrm{D}-04$ & $4.5406 \mathrm{D}-03$ & $3.3875 \mathrm{D}-03$ \\
2 & $1.4860 \mathrm{D}-05$ & $1.0659 \mathrm{D}-07$ & $4.2915 \mathrm{D}-09$ \\
3 & $1.2217 \mathrm{D}-09$ & $5.3618 \mathrm{D}-17$ & $4.6065 \mathrm{D}-13$ \\
4 & $3.2799 \mathrm{D}-12$ & $2.9704 \mathrm{D}-19$ & $8.9802 \mathrm{D}-16$ \\
5 & $3.5384 \mathrm{D}-15$ & $9.6265 \mathrm{D}-20$ & $5.5209 \mathrm{D}-18$ \\
6 & $1.0658 \mathrm{D}-17$ & $4.2631 \mathrm{D}-20$ & $1.1014 \mathrm{D}-18$ \\
7 & $1.3431 \mathrm{D}-18$ & $3.2871 \mathrm{D}-20$ & $2.0555 \mathrm{D}-19(\lambda)$ \\
8 & $2.8892 \mathrm{D}-19$ & $3.2616 \mathrm{D}-20$ & $9.5000 \mathrm{D}-19(\lambda)$ \\
9 & $2.9116 \mathrm{D}-19$ & $3.2613 \mathrm{D}-20$ & \\
10 & $1.0835 \mathrm{D}-19$ & & $9.3647 \mathrm{D}-20(\lambda)$ \\
11 & $1.1456 \mathrm{D}-18$ & Converged to & \\
& $1.0816 \mathrm{D}-19$ & $\varepsilon=1 . \mathrm{D}-4$ & $9.0576 \mathrm{D}-20(\lambda)$ \\
$\ldots$ & Terminated due & & \\
19 & to no further & & $6.9932 \mathrm{D}-20(\lambda)$ \\
\hline
\end{tabular}

An efficient practical approach would be to hybridize the integration and optimization methods, i.e., switch between the different methods as the optimization progresses. For example, in the case of rigid-body aerodynamics, it would be appropriate to start with a second-order Runge-Kutta integration and Gauss-Newton method. After a few iterations the fourth-order Runge-Kutta can be switched on. The saving in computational time, although marginal for small problems, could be considerable for global model identification requiring processing large amount of flight data with six degrees-of-freedom model.

\section{Estimation accounting for process noise}

The most commonly applied output error method yields biased estimates when random process noise excites the system, for example, flight data gathered in the presence of atmospheric turbulence. In such cases filter error methods are necessary (Maine \& Iliff 1981; Jategaonkar \& Plaetschke 1989). Figure 1 shows that even in the case of flight maneuvers in smooth air, filter error methods could lead to better estimation of results, because some of the unavoidable modelling errors are then treated as process noise characterized by lowfrequency contents rather than as measurement noise (Maine \& Iliff 1981; Jategaonkar 1993).

The C-160 data analysed in figure 1 were gathered from eight flights carried out during a span of less than two weeks, seven of them being in a seemingly steady atmosphere, while one encountered moderate turbulence. It is clearly visible that the estimates provided by the output error method, particularly those for flight 223 during which moderate turbulence was encountered, differ much from those of other flights at the same nominal flight conditions 
$C_{n \beta}$ (a)

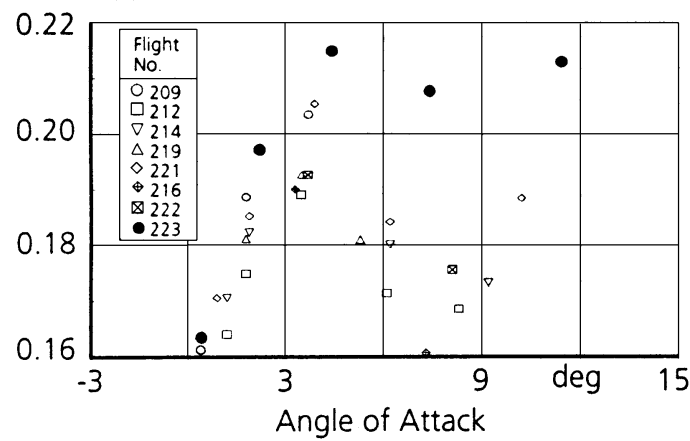

(b)

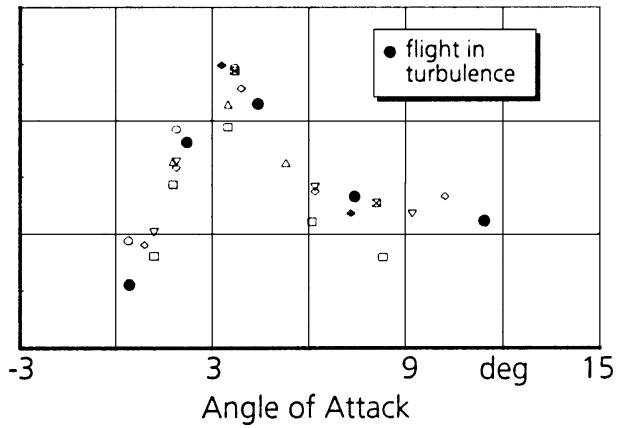

Figure 1. Flight estimates of weathercock stability by the output error (a) and filter error (b) methods.

(Jategaonkar 1993; Hamel \& Jategaonkar 1996). Moreover, a fair amount of scatter is observed in the estimates from other flights in a seemingly steady atmosphere, making the final conclusion regarding the nature of the nonlinearity or fairing of data difficult. On the other hand, the filter error method yields clearly grouped estimates with much less scatter and estimates from flight 223 match well with other estimates. The nonlinear dependency of the weathercock stability on the angle of attack is now observed more clearly.

\section{Unstable aircraft identification}

The demands of high performance characteristics have led to aerodynamically unstable aircraft configurations. Although unstable aircraft can be flown only with the aid of a flight controller, i.e. in a closed loop, determination of the aerodynamic characteristics of the basic aircraft, i.e. of the open-loop plant, is of primary interest in several instances.

The simplest approach to identification of unstable aircraft is to use linear regression in the time domain or any estimation method in the frequency domain. Application of the other time-domain methods to such cases, however, needs some consideration. The most widely used output error method in this case encounters numerical difficulties of diverging solution. Some special techniques and modifications are hence necessary to prevent the growth of errors introduced by poor initial values, round-off or discretization and propagated by inherent instabilities of the system equations. Several solutions such as: (i) S-plane transformation, (ii) output error method with artificial stabilization, (iii) equation decoupling, (iv) a relatively new approach called multiple-shooting based on efficient techniques for the solution of two-point boundary value problems, and (v) parameter estimation by filtering approach using extended Kalman filters, are possible (Hamel \& Jategaonkar 1996). These approaches, although providing solutions in particular cases, were found to involve engineering judgement, or required considerable effort, or the results could not be completely resolved. On the other hand, the filter error method and the regression method appear to be more readily applicable to unstable aircraft. The filter error method may have some advantages, particularly in the presence of considerable measurement noise, in which case the regression analysis yields biased estimates. In any case, a method which accounts for process noise is preferable, since the controller feeds back the measured variables containing measurement noise, and thereby introduces a component of stochastic input. 


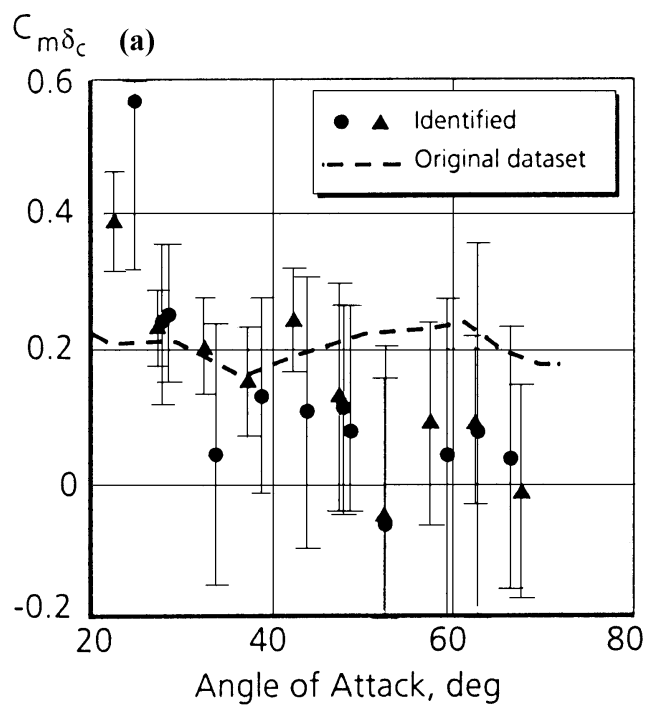

(b)

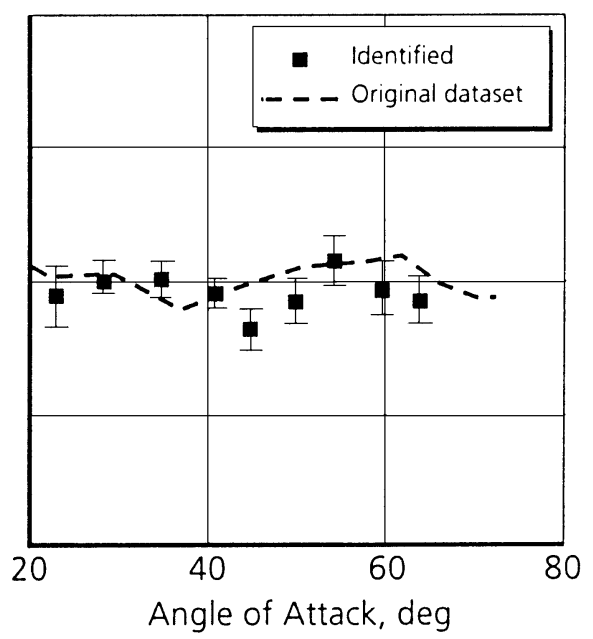

Figure 2. Estimates of canard control effectiveness from X-31A flight data pilot input (a) and separate surface excitation (b) maneuvers.

Apart from the choice of a suitable method, yet another serious difficulty encountered in unstable aircraft identification is that of parameter identifiability. The controller tends to suppress oscillatory and transient motion, which is what it is anyway designed for. It is, however, detrimental to the identifiability and accuracy of the parameter-estimates, since the information content in the data is drastically reduced. Furthermore, the feedback results in correlated inputs and also correlated motion variables. The combined solution to both these problems is to introduce controlled inputs directly deflecting off the control surfaces. This is often called in the literature as Separate Surface Excitation (SSE). Even in the case of SSE inputs the feedback controller would be active; however, the initial aircraft response to SSE-input is characteristically different and less correlated compared to that due to pilot input.

As a typical example, figure 2 shows the estimates of the canard control effectiveness obtained from the X-31A flight test data for two cases, namely pilot input maneuvers and separate surface excitation (Weiss et al 1995). As evident from figure 2a, pilot input maneuvers yield estimates with large standard deviations shown by vertical bars; moreover, the scatter is also large. This is definitely attributed to the aforementioned difficulties of insufficient information content and correlated variables. On the other hand, the separate surface excitation maneuvers yield well-identifiable estimates shown in figure $2 \mathrm{~b}$. Thus, separate surface excitation greatly eliminates the problems due to correlated inputs and motion variables. Separate surface excitation is, however, a complex procedure requiring hardware modifications and often flight certification. Otherwise, the alternative approach would be to attempt parameter-estimation based on data-collinearity and mixed estimation. In such cases, however, it may be possible to obtain unbiased estimates of only a subset of parameters. Moreover, the basic problem of insufficient excitation still persists. Although the aspects of parameter identifiability and data-collinearity have been discussed in the context of unstable aircraft, these issues are equally applicable to stable aircraft as well.

Identification of open-loop unstable aircraft via closed-loop identification, although feasible, is rather impractical. From such an attempt, to obtain the open-loop parameters of 
the basic aircraft would require incorporating the models for the controller and actuator dynamics in the estimation procedure. The overall system being stable, any standard parameter estimation method can be applied without encountering any serious difficulty. With the current state of the art, even the increased model size should not be a serious problem. The primary difficulty is to obtain the exact models for the complex control laws containing discrete nonlinearities. In addition, actuator performance and controller gains may be flight condition dependent. Moreover, this approach may result in open-loop parameter estimates with low accuracy.

\section{Aerodynamic databases for high fidelity flight simulators}

Estimation of a comprehensive aerodynamic model suitable for a flight simulator is an iterative process, which starts with point identification at all the points flight tested. Pointidentification results in a model related to specific trim conditions. Based on this bulk of estimation results, aerodynamic model postulates can be extended to include angle-ofattack or Mach number dependencies, coupling derivatives, and other nonlinearities. Through multi-point identification, several flight conditions can be analysed simultaneously to arrive at a comprehensive model. This general approach was followed in the generation of aerodynamic databases for DLR's in-flight simulator ATTAS (Jategaonkar 1990), military transport aircraft C-160 (Jategaonkar et al 1994) and commuter aircraft Dornier 328 (Jategaonkar \& Mönnich 1997). As a typical example, some details of C-160 data gathering are provided in this paper.

The complete data gathering program, carried out with the instrumented aircraft, figure 3 , consisted of flight tests for: (i) Calibration of air data system, (ii) estimation of aerodynamic database, (iii) ground tests (taxiing, ground acceleration/deceleration, stopping distances), (iv) validation tests for simulator certification according to FAA guidelines, (v) sound data, (vi) C-160 specific operational characteristics (load drop, landing and takeoff on unprepared terrain and short field, single engine flights), and (vii) stall dynamics. Altogether 28 flights were carried out, totalling 79 flight hours. A total of 350 trim

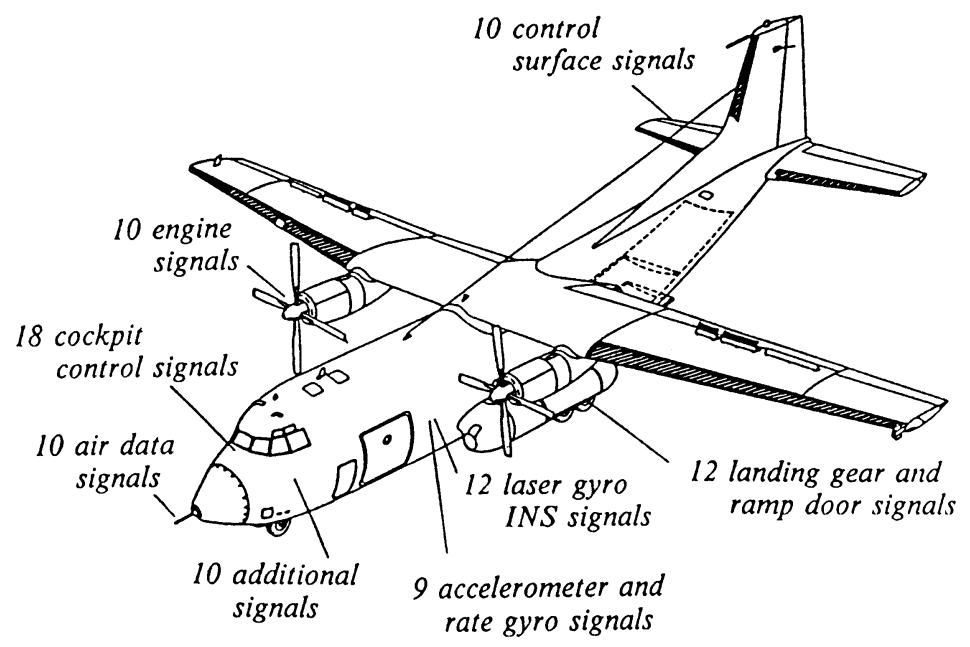

Figure 3. Instrumented C-160 "Transall” aircraft. 


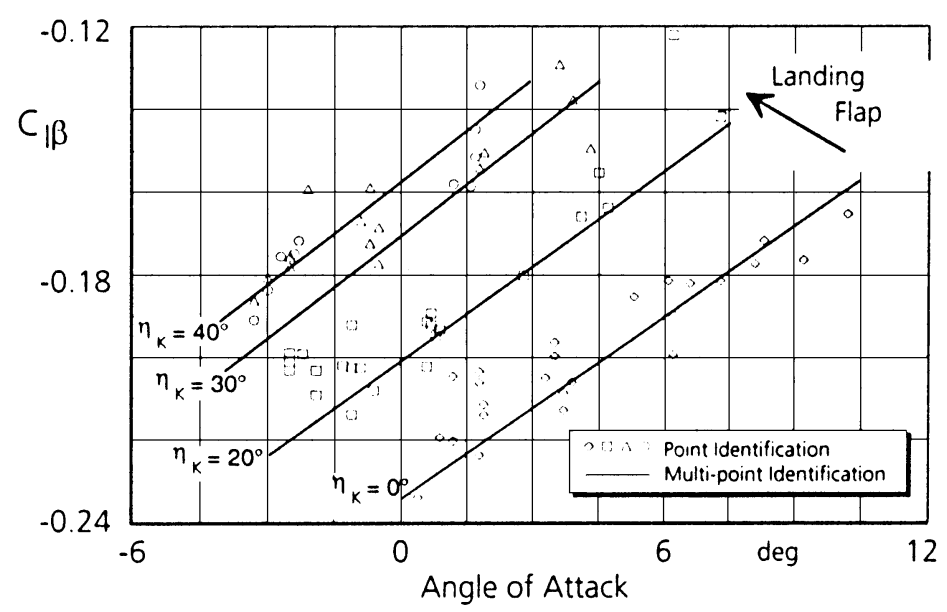

Figure 4. C-160 dihedral effect (derivative $C_{l \beta}$ ).

conditions and 964 system-identification maneuvers including 22 stall maneuvers were investigated covering altitudes up to $26000 \mathrm{ft}(\approx 8000 \mathrm{~m})$ and Mach numbers up to 0.52. Test points were judiciously chosen to span the entire operational envelope and possible configurations: (i) Clean configuration (flap $\eta_{K}=0^{\circ}$, nominal CG of $28 \% \mathrm{MAC}$ ), (ii) landing flaps $\left(\eta_{K}=20^{\circ}, 30^{\circ}, 40^{\circ}, 60^{\circ}\right)$, (iii) CG locations (forward $23 \%$, nominal $28 \%$, and aft $33 \%$ MAC), (iv) landing gear, (v) ramp door, and (vi) one engine out.

A detailed description of aerodynamic modelling and all other pertaining issues is provided by Jategaonkar et al (1994). For illustration, the estimates of the dihedral effect, derivative $C_{l \beta}$, obtained by point-identification, are shown for four landing flap positions in figure 4 . The estimates are clearly a function of angle of attack and also of landing flap position. The same figure shows the multi-point identification results for each flap position, obtained by analysing several flight maneuvers simultaneously.

In general, determination of primary derivatives does not pose any serious difficulties. For high-fidelity databases for flight simulators, it is, however, necessary to properly model the aerodynamic effects of secondary order as well. This is particularly true when the model fidelity has to meet the level D standards specified by the FAA. The ramp door and landing gear effects, or the downwash due to speed brakes, are some typical examples of this class (Jategaonkar et al 1997).

Figure 5 shows two flight maneuvers flown with extended landing gear; the first maneuver is a short period motion excited by a 3211 input and the second is an aileron and rudder input maneuver resulting in rolling and yawing motion. The basic aerodynamic model identified from the clean configuration, i.e. neglecting the landing gear effects, yields the response match shown in figure 5a, where $a_{x}$ is the longitudinal acceleration, $r$ is the yaw rate, $\theta$ is the pitch attitude, $\alpha$ is the angle of attack, and $\beta$ is the angle of sideslip. Discrepancies in the longitudinal acceleration are demonstrative of the additional drag being introduced. The lateral-directional variables, angle of sideslip $\beta$ and yawing rate $r$, also show some discernible differences.

Through a systematic approach, and keeping in mind the principle of parsimony, additional parameters were identified to model the incremental effects due to landing gear (Jategaonkar et al 1997). The identified value of $\Delta C_{\mathrm{DLG}}$, the incremental change in the drag coefficient $C_{\mathrm{D}}$ due to landing gear (subscript LG), suggests increased drag and the 

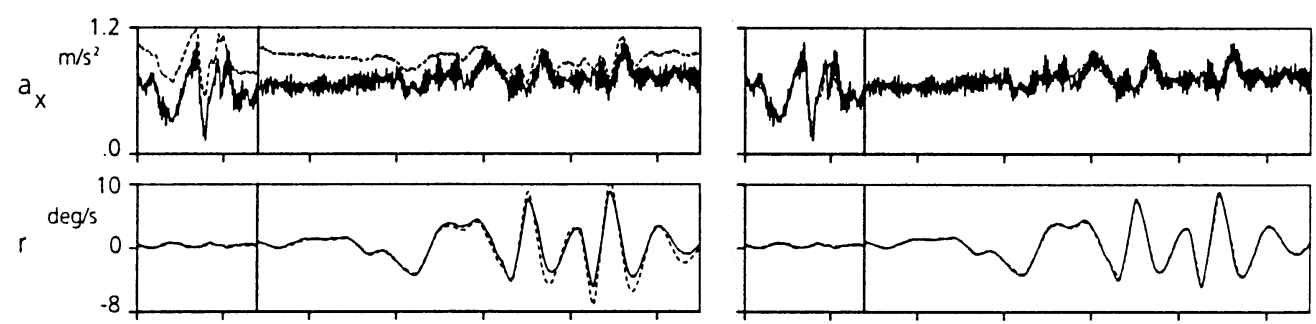

$\theta$
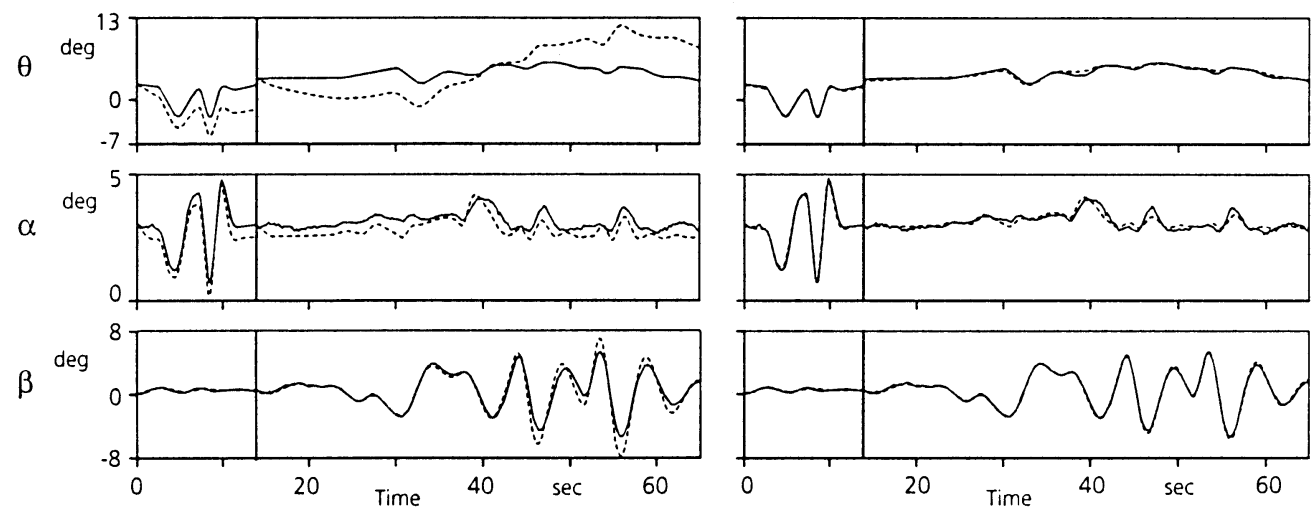

(a)

(b)

Figure 5. Estimation of aerodynamic effects of secondary order-C-160 landing gear effects (- flight measured; - - - - - estimated), obtained neglecting (a) and accounting for (b) landing gear effects.

derivative $C_{n \beta \mathrm{LG}}$, increased weathercock stability. The resulting model yields the response match shown in figure $5 \mathrm{~b}$, which is significantly better compared to that in figure 5a. Landing gear effects are typically in the range of $5 \%$ to $10 \%$ of the total, but are still important for simulator fidelity. Proper test techniques and a systematic modelling approach enable accurate identification of such aerodynamic effects of secondary order.

\section{Material parameter identification of stochastic constitutive models}

A related application of the developed algorithms and software tools for aircraft parameter estimation is the modelling of elasto-viscoplastic deformations of metallic materials. Predictions of mechanical behaviour play a central role in the design and analysis of many technical components and structures in aircraft systems, power generation systems and several other industrial applications. An important phenomenon is the time-dependent inelastic deformation even under constant load. In particular, for long operation times of technical components, description of the creep processes in material models is of high importance.

A lot of mathematical models, also called constitutive equations, have been developed in the past covering a wide spectrum of representations. Modern constitutive models mostly describe not only the macroscopically observed deformation but also the evolution of the internal material structure. The basic mechanism which leads to inelastic deformations are movements of dislocations. Investigations concerning the development of material models based on mechanisms on the micro scale have resulted in a unified stochastic model which is able to represent essential and typical features of the low and high temperature plasticity. 
For the stochastic modelling of dislocation movements in crystalline materials and their temperature $T$ and stress $\sigma$ activation, a discrete Markov chain is considered. In order to describe cyclic material behaviour, we use the widely accepted concept that the dislocation gliding processes are driven by the effective stress $\sigma_{\text {eff }}$ as the difference between the applied stress $\sigma$ and the internal back stress $\sigma_{\text {kin. }}$. The influence of effective stress and temperature on the inelastic deformations considered in the evolution equations is based on the physical properties of the metal. It can be shown that the mean value formulation of the stochastic model leads to a nonlinear system of differential equations for the inelastic strain $\varepsilon_{\text {ie }}$, the kinematic back stress $\sigma_{\text {kin }}$, and the activation volume $V$. In the low temperature regime, hardening is the most important phenomenon for the development of the material structure while the recovery influence is negligible. Thus, considering the influence of the effective stress which can be modelled by a sinus hyperbolic function, the viscoplastic behaviour, for example of copper, can be modelled as (Thielecke 1996):

$$
\begin{aligned}
\dot{\varepsilon}_{\text {ie }} & =C \cdot \exp \left(-\frac{F_{0}}{\mathcal{R} T}\right) \cdot \sinh \left(\frac{V \sigma_{\text {eff }}}{\mathcal{R} T}\right), \\
\dot{\sigma}_{\text {kin }} & =H \cdot \exp \left(-\frac{\delta V \cdot \sigma_{\text {kin }}}{\mathcal{R} T} \cdot \operatorname{sign} \sigma_{\text {eff }}\right) \cdot \dot{\varepsilon}_{e}, \\
\dot{V} & =-K_{1} V^{2} \cdot\left|\dot{\varepsilon}_{\text {ie }}\right|+K_{2} V \cdot\left|\dot{\varepsilon}_{\text {ie }}\right| .
\end{aligned}
$$

The constitutive equations contain several unknown parameters $\left(C, H, \delta, K_{1}, K_{2}\right)$ characterizing the material properties that have to be identified from experimental data. Since the general form of the stochastic model must be adapted to the special material characteristics and the considered temperature regime, identification of the unknown material parameters plays an important role for the application based on numerical calculations. Compared to flight vehicle system identification, the current problem has the following properties:

- The constitutive model is highly nonlinear in states and parameters and may become stiff.

- Experimental data are available only for the strain rate, no observation can be obtained for the internal states.

- The identification problem is ill-conditioned.

- The measurements are incompatible due to scattering.

The determination of the unknown material parameters is based on a maximum-likelihood output-error method comparing the experimental data with the numerical simulations. For the minimization of the cost function, the hybrid optimization concept is considered. It couples stochastic search procedures and several Newton-type methods. A relatively new approach for material parameter estimation is the multiple-shooting approach which allows efficient use of additional measurement and a priori information about the states. This reduces the influence of bad initial parameters.

A lot of different materials like pure aluminum, pure copper or austenitic steels AISI 304 and AISI 316 have been extensively studied (Thielecke 1998). Figure 6 shows the typical example of copper, showing the simulated and measured stress-strain curves in figure $6 \mathrm{a}$ and the corresponding time histories of stress in figure $6 \mathrm{~b}$. The experimental data from seven strain-controlled cyclic tests at room temperature are examined. Two strain rates of $\dot{\varepsilon}=10^{-4} 1 / \mathrm{s}$ and $\dot{\varepsilon}=10^{-3} 1 / \mathrm{s}$, and a multitude of strain amplitudes $(\Delta \varepsilon / 2=0.2-0.7 \%)$ 


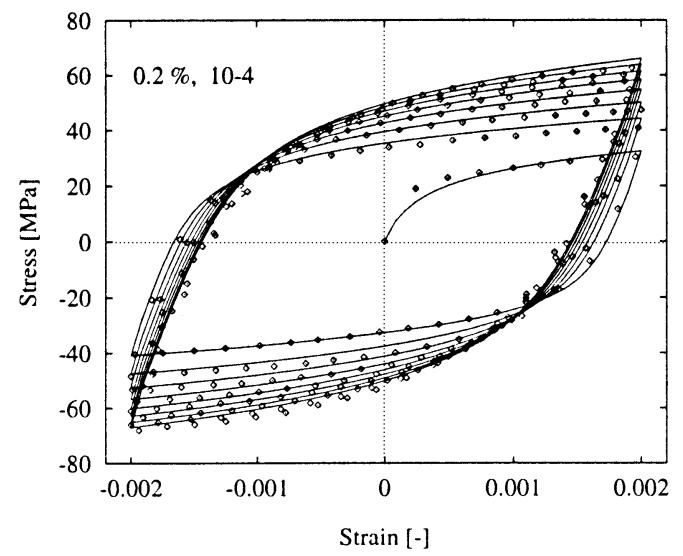

(a)

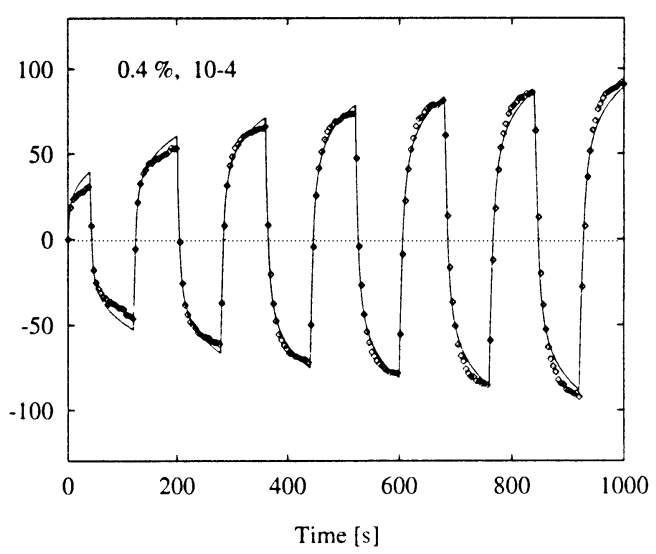

(b)

Figure 6. Cyclic tests for copper: comparison of simulated and measured stress and strain ( $\circ \circ \circ \circ \circ \circ \circ \circ \circ$ measured; - estimated). (a) Stress-strain curve; $\Delta \varepsilon / 2=0.2 \%$, $\dot{\varepsilon}=10^{4} 1 / \mathrm{s}$; (b) stress time history; $\Delta \varepsilon / 2=0.4 \%, \dot{\varepsilon}=10^{-4} 1 / \mathrm{s}$.

are analysed. For this application of the stochastic constitutive model, the special characteristics of the material and the measurements are that cyclic hardening can be observed as also the Bauschinger-effect, which can be recognized by the fact that plastic flow occurs after load reversal at significantly lower stresses than those at which the load reversal was done. For the technical use of metallic materials, the description of this kind of process in material models is of high importance.

The statistical analysis of the estimates shows that in general all parameters are estimated quite well, see table 3. Some parameter pairs, however, show somewhat higher correlation, for example, the estimates of $C$ and $H$ having a correlation coefficient of 0.95 , and $K_{1}$ and $K_{2}$ with 0.91 , see table 4 . Parameter estimation by analysing more experimental data from different types of tests can lead to better estimates with reduced correlation.

Figure 7 shows some results of parameter estimation for aluminum Al 99.999. The temperature regime is between $500^{\circ}$ and $700^{\circ} \mathrm{C}$. Since only monotonic tests are evaluated, a constitutive model with only one structure variable for the internal stress is used. The parameters are identified for the given stresses simultaneously, so that the calculated curves are obtained by a single parameter set.

Experimental data to determine the parameters of constitutive equations usually consist of only one observed trajectory for each temperature and loading condition. Nevertheless replicated tests for the same laboratory conditions show a significant scattering and thus incompatibility of the measurements. Based on a statistical analysis this uncertainty

Table 3. Parameter estimates and standard deviation.

\begin{tabular}{llcc}
\hline Parameter & Estimate & Absolute standard deviation & Relative standard deviation $\%$ \\
\hline$C$ & $1.24 \times 10^{4}$ & $\pm 5.89 \times 10^{3}$ & 47.92 \\
$H$ & 1.19 & $\pm 2.36 \times 10^{-1}$ & 19.98 \\
$\delta$ & $4.30 \times 10^{-1}$ & $\pm 7.98 \times 10^{-3}$ & 1.87 \\
$K_{1}$ & $8.63 \times 10$ & \pm 3.30 & 3.90 \\
$K_{2}$ & $2.43 \times 10$ & \pm 8.29 & 3.50 \\
\hline
\end{tabular}


Table 4. Correlation amongst the parameter estimates.

\begin{tabular}{lccccc}
\hline & $C$ & $H$ & $\delta$ & $K_{1}$ & $K_{2}$ \\
\hline$C$ & 1.00 & & & & \\
$H$ & 0.95 & 1.00 & & & \\
$\delta$ & 0.25 & 0.24 & 1.00 & & \\
$K_{1}$ & 0.32 & 0.25 & 0.71 & 1.00 & \\
$K_{2}$ & 0.34 & 0.30 & 0.46 & 0.91 & 1.00 \\
\hline
\end{tabular}

can be taken into account for more reliable modelling and parameter estimation, see figure 8 .

Modelling of the experimental uncertainties is based on the scattering of the parameters or the initial values. Based on these concepts, realistic simulations of the uncertainties in experimental data due to measurement errors and scattering are possible, see figure 9.

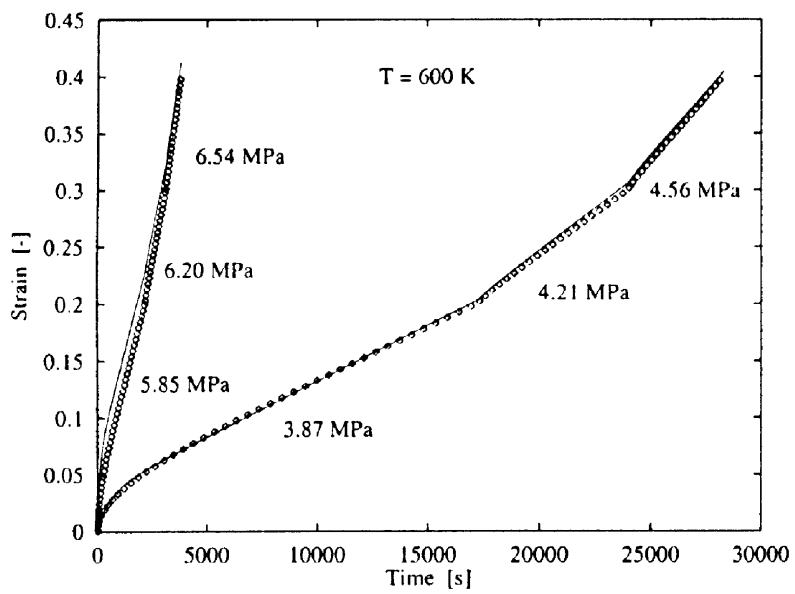

(a)

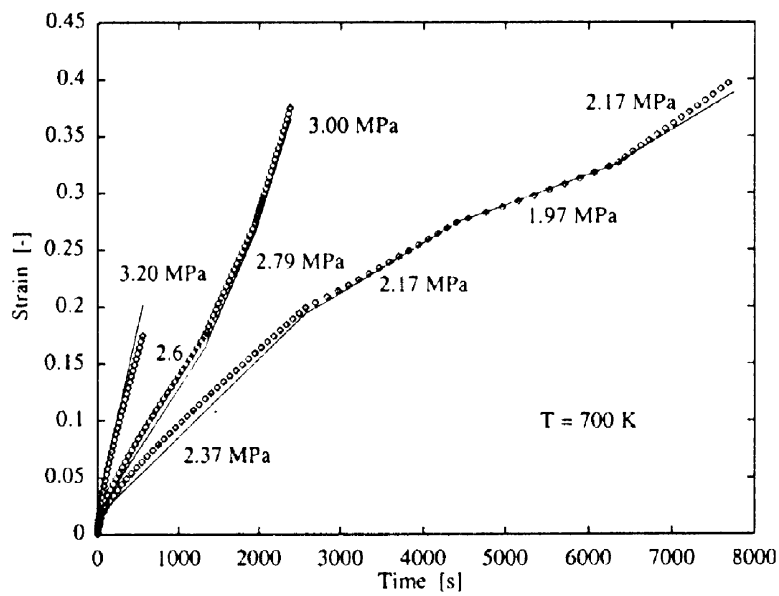

(b)

Figure 7. Creep test for aluminum: comparison of simulated and measured strain ( $\circ \circ \circ$ measured; - estimated) at temperature $T=600 \mathrm{~K}$ (a) and $700 \mathrm{~K}(\mathbf{b})$. 


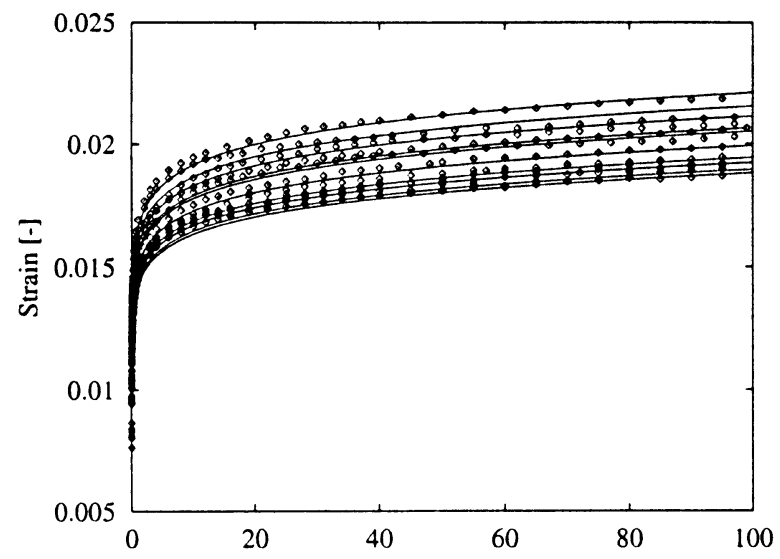

(a)

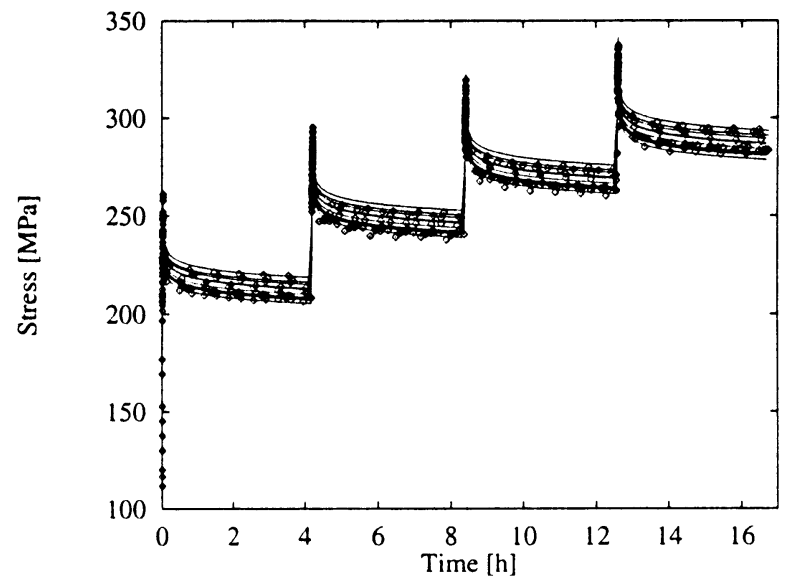

(b)

Figure 8. Scattering of creep and tension-relaxation tests for AISI 316 ( $\circ \circ \circ$ measured; —estimated).

(a) Strain and (b) stress.
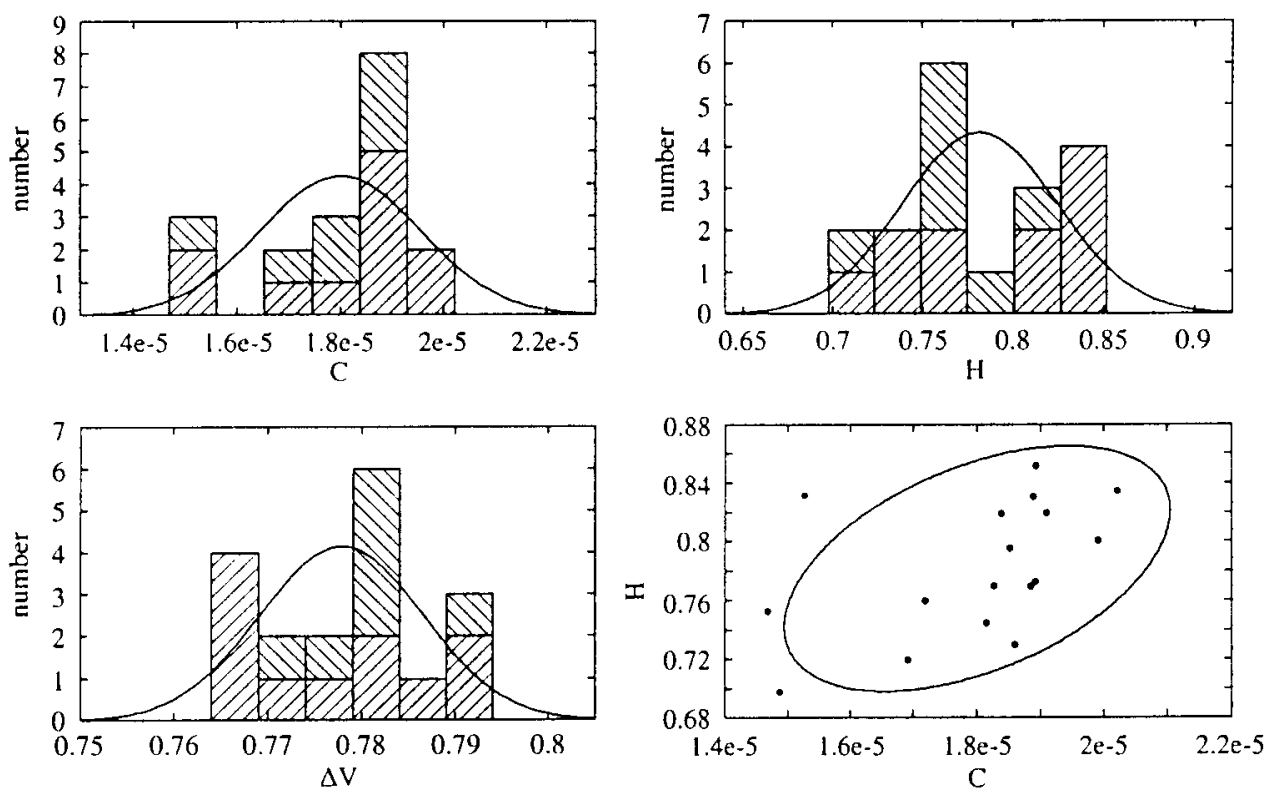

Figure 9. Probability density function and correlation of scattered parameters. 


\section{Summary}

The paper demonstrates successful application of the system identification methodology to a variety of problems in flight mechanics and also to modelling of inelastic deformations of metallic materials. Practical aspects of handling nonlinear systems, computation of gradients, and the choice of integration and optimization methods are brought out. Issues related to unstable aircraft and estimation accounting for atmospheric turbulence are elucidated. Based on the typical examples presented in the paper, it can be concluded that parameter estimation methods provide an efficient and powerful tool to generate flight validated, high-fidelity aerodynamic databases. These tools have also proved useful in related applications, such as modelling of metallic strain-stress characteristics.

\section{References}

Anon 1991 Airplane simulator qualification. FAA Advisory Circular AC 120-40B, Interim Version Anon 1995 Airplane flight simulator evaluation handbook (London: Royal Aeronaut. Soc.)

Chevalier H L, Dornfeld G M, Schwanz R C 1968 An analytical method for predicting the stability and control characteristics of large elastic airplanes at subsonic and supersonic speeds-Part II: Application. NASA TM-X-66378

Hamel P G 1981 Determination of aircraft dynamic stability and control parameters from flight testing. AGARD LS-114, paper no. 10

Hamel P G, Kaletka J 1995 Rotorcraft system identification - An overview of AGARD FVP Working Group 18. AGARD CP-552, paper no. 18

Hamel P G, Jategaonkar R V 1996 Evolution of flight vehicle system identification. J. Aircraft 33: 9-28

Hamel P G, Jategaonkar R V 1998 The role of system identification for flight vehicle applications Revisited. RTO-MP-March 1999, pp 2.1-2.12

Huber P 1995 Control law design for tailless configurations and in-flight simulation using the X-31 aircraft. AIAA Guidance, Navigation, and Control Conference, Baltimore, MD, paper no. 95-3199

Jategaonkar R V 1990 Identification of the aerodynamic model of the DLR research aircraft ATTAS from flight test data. DLR-FB 90-40, July 1990

Jategaonkar R V 1993 A comparison of output error and filter error methods from aircraft parameter estimation results. DLR-Mitt. 93-14, pp 63-87

Jategaonkar R V, Mönnich W 1997 Identification of DO-328 aerodynamic database for a level D flight simulator. AIAA Atmospheric Flight Mechanics Conference (Washington, DC: AIAA) paper 97-3729

Jategaonkar R V, Plaetschke E 1989 Algorithms for aircraft parameter estimation accounting for process and measurement noise. J. Aircraft 26: 360-372

Jategaonkar R V, Thielecke F 1994 Evaluation of parameter estimation methods for unstable aircraft. J. Aircraft 31: 510-519

Jategaonkar R V, Mönnich W, Fischenberg D, Krag B 1994 Identification of C-160 simulator data base from flight data. Proceedings of the 10th IFAC Symposium on System Identification, Copenhagen, Denmark, vol. 3, pp. 3.67-3.74

Jategaonkar R V, Mönnich W, Fischenberg D, Krag B 1997 Identification of speed brake, air-drop, and landing gear effects from flight data. J. Aircraft 34: 174-180

Klein V 1989 Estimation of aircraft aerodynamic parameters from flight data. Progress in aerospace sciences (Oxford: Pergamon) vol. 26, pp 1-77

Maine R E, Iliff K W 1981 Formulation and implementation of a practical algorithm for parameter estimation with process and measurement noise. SIAM J. Appl. Math. 41: 558-579

Maine R E, Iliff K W 1986 Identification of dynamic systems - Applications to aircraft. Part 1: The output error approach. AGARD AG-300- vol. 3, part 1 
Marquardt D W 1963 An algorithm for least-squares estimation of nonlinear parameters. J. Soc. Ind. Appl. Math. 11: 431-441

Ross H 1991 X-31 enhancement of aerodynamics for maneuvering beyond stall. AGARD CP-497, paper no. 2

Rynaski E G, Andrisani D II, Weingarten N C 1978 Identification of the stability parameters of an aeroelastic airplane. AIAA 78-1328

Thielecke F 1996 Numerical concepts for material parameter identification of a stochastic constitutive model. Proceedings of ECCOMAS 96 (New York: John Wiley \& Sons)

Thielecke F 1998 Parameteridentifizierung von Simulationsmodellen für das viskoplastische Verhalten von Metallen. Ph D thesis, Technical University of Braunschweig, Braunschweiger Schriften zur Mechanik no. 34-1998

Weiss S, Friehmelt H, Plaetschke E, Rohlf D 1995 X-31A system identification using single surface excitation at high angles of attack. AIAA paper 95-3436 